\title{
Breaking a Diagnosis of Dementia
}

\author{
M. Dalvi \\ William Harvey Hospital, Ashford Kent \\ Kent EMedway NHSE Social Care Partnership Trust, Kent, \\ UK
}

\section{Introduction}

There are 700,000 people in the UK living with Dementia (Knapp etal, Dementia UK: The full Report Alzheimer's Society2007). This has prompted the government to publish the first National Dementia Strategy which has made dementia a national priority. Dementia has a huge impact on physical, psychological and social aspects of care and also poses ethical challenges. One major ethical challenge which looms large, is the difficulty faced by clinicians in disclosing the diagnosis of dementia. The National Dementia strategy acknowledges this. 61\% General practitioners routinely withhold diagnosis (Vassilas C\& Donaldson J, 1998) and 60\% Old Age Psychiatrists do not always disclose diagnosis as $20 \%$ do not see any benefit (Downs et al, 2002). This is similar to disclosure practices with cancer patients in the 1960 's whereby $90 \%$ of physicians did not disclose the diagnosis of cancer to their patients (Oken etal 1961). This however saw a fundamental shift in the seventies when only $10 \%$ of physicians withheld the diagnosis of cancer (Novack etal, 1979). This may be related to new cancer medications and patient rights. There are many reasons for not disclosing a diagnosis of dementia. Physicians fear causing harm to their patients. (Pinner G\& Bouman W, 2002). Some medical practitioners find it hard to share a diagnosis of dementia( Illife,S etal,03) and others report explaining a diagnosis of dementia is more difficult than for other diseases( Glosser etal,1985). This can lead to variability in diagnostic disclosure which I will address here. Since the last decade great progress has been made in identifying biomarkers and molecular changes in the brain associated with Alzheimer's disease. With various disease modifying medications like Bapineuzumab in phase III trials showing promising results, diagnostic disclosure is finding itself in the limelight for ethical and scientific reasons.

\section{Problems with diagnosis of dementia}

There are inherent problems with diagnosing dementia itself due to the insidious nature of the condition and difficulty in detecting transition between normal ageing and the onset of dementia. Diagnosis is based on clinical criteria and takes into account history, physical and neurological examination along with appropriate laboratory investigations. Due to the lack of a single and definitive tool for diagnosing Alzheimer's disease and other forms of dementias, one can easily miss out on early diagnosis. A definitive diagnosis of dementia is only possible at post-mortem. Several diagnostic tools are available with their own unique problems. The widely used Mini-mental state exam had demonstrated less sensitivity to 
mild dementia in highly educated people and those with non-cortical dementias. (Rothlind \& Brondt, 1993). The NINCD-ADRDA criteria (McKhann etal, 1984) have a high degree of sensitivity but low specificity for the diagnosis of possible Alzheimer's disease (Knopman etal, 2001). This may lead to over diagnosis of some patients without dementia. All this can lead to diagnostic uncertainty and when coupled with therapeutic nihilism may lead to resistance in the disclosure of diagnosis. Although there is a substantial body of evidence which favours diagnostic disclosure there is a huge variability in all the aspects of disclosure (Carpenter\& Dave, 2004). Bamford and colleagues concluded from their meta-analysis that the process of disclosure is not easy. People with dementia are less often told of their diagnosis than their family members. There is widespread use of euphemisms and family members generally prefer not to inform the diagnosis to their relatives.

\section{Ethical principles in diagnostic disclosure}

According to duty based ethics developed by the German philosopher Emmanuel Kant, it is morally wrong not to disclose the diagnosis even if it has harmful consequence. This may directly contradict the ethical principle of non-maleficence i.e. avoiding harm. So there is an ethical dilemma as to whether all treatments and interventions which may lead to harm whether psychological or in the form of side-effects should be avoided on the grounds that "avoiding harm always takes priority over doing good".

Autonomy: Patients have the right to think, decide, and act on the basis of such thoughts, freely and independently. Clinicians are faced with a dilemma of respecting patients' autonomy on one hand and concerns of carers at the same time. This has now been clarified by the Nuffield Council of Bioethics in its report Dementia: Ethical issues (Nuffield Council of Bioethics .Dementia: Ethical issues, Oct 2009). It suggests a broad concept of autonomy called "Relational autonomy" for patients suffering from dementia. (Nuffield Council of Bioethics. Dementia: ethical issues.2009). It suggests that as patient's sense of self is grounded in their social network, the whole family should be helped to support the autonomous wishes of the patient. This concept in my view is very helpful as on one hand it helps to maximise patients' freedom and on the other hand helps to minimise risk. It also recommends that clinicians should actively encourage patients to share details about their illness with their family.

General Medical Council recommends that doctors must give their patients information they request for. In practice, patients with dementia rarely ask for information, so should we hide the diagnosis if they don't ask for it? Is this ethical?

\section{Current practice}

Views of consultants, patients and family members in sharing the diagnosis are different (McWilliams, 1998) Patients prefer to be told of their diagnosis (George\& Gove, 2007) however in reality things are different. Only half of geriatricians openly discuss the diagnosis with their patients (Carpenter\&Dave, 2004). Family members generally prefer not to inform people with dementia, despite agreeing that they would want to know the diagnosis if they were in that situation. (Bamfordetal, 2004).

Keady\& Gilleard (2002) report that the experience of patients about assessment and disclosure is negative. Patients' perceived it as a controlling, insensitive process with feelings of insecurity, uncertainty and anxiety. Delays in assessments in the memory clinic was unsettling and patients felt stigmatised to the diagnosis and location of the memory 
clinic. (Pratt\& Wilkinson, 2001) report that patients had feelings of shock, anger and denial after receiving the diagnosis. They felt pressured to perform well on the memory tests because of drug therapy. Patients were concerned about late disclosure of diagnosis and lack of information on prognosis.

Reasons against diagnostic disclosure: are largely based on the principle of non-maleficence (Drickamer etal, 1992). This is due to the following reasons

1. concerns for causing harm, distress to patients i.e. Therapeutic lying (Bakhurst,1992)

2. lack of definitive diagnosis

3. No curative treatment.

4. Concerns about ability to understand information in advanced dementia.

5. No benefits, costs outweigh benefits.

6. Stigma associated with dementia.

Reasons for diagnostic disclosure are based on the ethical principle of autonomy.

1. Patient has the right to honest information, to know about their diagnosis according to the above principle. Hiding diagnosis will breach autonomy.

2. Several studies confirm that patients with dementia prefer to be informed of the diagnosis of dementia(Erde etal,1988 Maranski,2000.,Clare,2003.,Van Hout etal,2006)

3. Non disclosure can upset, confuse patients and break trust( Bamford etal,2004)

4. Patients feel relieved after diagnosis( Derksen etal,2006)

5. It helps patients plan for the future.

6. For travel and vacation purposes.

7. Obtaining a second opinion.

There is more evidence base for diagnostic disclosure as opposed to non- disclosure. Patients prefer to be informed of the diagnosis and are distressed if they are not informed of their diagnosis (Clare, 2003; Pratt\& Wilkinson, 2003). The work done by (Jha etal, 2001) emphasises that there is no evidence that disclosing diagnosis leads to harm in the form of stigma, depression or suicide.

Criticisms of the studies. Most of the above studies are surveys of convenience samples which compromise validity of results. The questionnaires may not be valid and reliable and can lead to social desirability effect. The study done by Erde and colleagues has recruited younger mostly cognitively intact patients which is not a representative sample.

There are various models of diagnostic disclosure but one should embrace a person-family centred approach based on breaking bad news. It should be a gradual educative process involving discussions with both the patient and family maintaining the dignity of the patient.

\subsection{Impact of new medical technology on diagnostic disclosure}

There is a growing evidence base for use of MRI, CSF amyloid, tau assays in Early Diagnosis of Dementia Duara and colleagues (Duara etal, 2008) highlights the role of structural MRI in the early diagnosis of $\mathrm{AD}$. Medial temporal atrophy has consistently been shown to represent an early imaging feature of $\mathrm{AD}$ and to predict conversion from $\mathrm{MCI}$ to $\mathrm{AD}$. (DeCarli etal, 2007) CSF assays and PET amyloid tracer uptake are sensitive at the earliest stage of Alzheimer's disease. 18F PET and MRI are sensitive at the Mild cognitive impairment stage and continue to change well into the dementia stage. This will greatly improve accuracy of early diagnosis and hence will have an impact on diagnostic disclosure .This will end uncertainty of diagnosis especially at the Mild cognitive impairment stage and clinicians will become more confident in diagnostic disclosure. 


\subsection{Preparation for a diagnostic assessment}

Factors influencing diagnosis. (Bamford etal, 2004)

1. Disease factors

a. Severity of cognitive impairment.

b. Diagnostic uncertainty.

2. Clinician Factors

a. Beliefs and type of clinician

b. Age and time since clinician qualified.

c. Clinician's attitude to early diagnosis.

3. Patient Factors

1. Age of patient.

2. Patient's desire to be told.

3. Insight of patient

4. Personality of the patient

5. Emotional stability of patient

6. Comorbidity of patient.

4. IV) Carer Factors

a. Age of carer

b. Carer's desire for patient to be told of the diagnosis.

More research is needed as to which of the above factors strongly influences the clinician during diagnostic disclosure. There is an emerging body of evidence on how cultural and religious beliefs of doctors influence End of Life care decisions and similar studies regarding personal values, religious and cultural beliefs influencing diagnostic disclosure are necessary.

\section{Outcomes of disclosure for patient's with dementia}

1. Positive Outcomes.

a. End to uncertainty

b. Confirmation of suspicions

c. Increased understanding of problems

d. Access to support

e. Helps to develop positive coping strategies

f. Planning and short term goals

2. Negative Outcomes

a. Negative effects on self esteem and personhood

b. Preoccupation with Diagnosis

c. Anxiety about increasing disability.

d. Restricted activities

e. Crisis after diagnosis

f. Hyper vigilant state after diagnosis

These outcomes need to be discussed with patients following which there needs to be sufficient space for patient's emotions. Clinicians may find it particularly challenging to disclose diagnosis in uncertain cases with comorbid psychiatric disorders. or for patient's who have normal tests. Patients with premorbid high IQ may score within the normal range on neuropsychological assessments and this may prove challenging for the clinician breaking the diagnosis. 


\section{Pre assessment Counselling framework}

a. Consent \& Choice

b. Collaboration\& Control

c. The person and their context

a. Consent- The patient should be informed about the reasons for the referral, process of assessment and beyond, outcomes of their assessment, implications of receiving a diagnosis of dementia as one of the possible outcomes of assessment. Every competent patient is considered to be autonomous and hence has the right of control over his body and hence we should obtain consent for diagnostic disclosure. An important step here is to give all the information to the patient before the disclosure. Not giving sufficient information before and after the diagnosis is not ethical. Also if the patient is not offered enough information to make their decision, their consent may not be valid. Ability to give consent may depend on context and patients should be given a choice.

b. Promoting Control and Collaboration: Patients should know that they have a choice regarding diagnostic disclosure. Their preference regarding type of feedback and location of the feedback should be honoured to prevent distress. Patients should be encouraged to share their diagnosis with their family members keeping in line with the new concept of "Relational Autonomy".

c. The Person and their context- Current life difficulties and their own ideas about their difficulties should be explored. Things that are going well should also be looked into. Coping strategies should be looked into and past crisis and response to it need to be explored. Their support networks and perceptions of significant others should be explored. Prior experiences of people they know with memory problems should be discussed. Past experiences of psychiatric services should be explored.

\section{Post Diagnostic information}

1. Emotions should be explored. Depression, denial, Anxiety are common. Earlier emotional experiences may be experienced again.

2. Cognitive changes and its impact should be addressed. Information about general loss of information processing, loss of function of recognition which may trigger emotions like anxiety and fear should be explained.

3. Losses e.g. loss of identity, loss of social role should be explored.

4. Future impact on relationships and attachments should be explained- Importance of bonds and relationships for support should be explained.

5. Environment and its impact- Loss of sense of familiar places should be explained.

6. Information about services like cognitive stimulation groups, support groups, Alzheimer's Society café, Admiral Nurses, Intermediate care team should be provided.

\subsection{Case Study 1}

Andrew who is a retired engineer lives with his wife Joan who suffered from memory lapses for several years. They have an outpatient clinic appointment in hospital for assessment of her memory problems. Joan was assessed by the doctor and asked to wait in another room. John who was outside in the waiting area was called in all by himself and the news that his wife has Alzheimer's disease was broken to him and the symptoms were confirmed.

Andrew was in the room with 3 strangers who sat looking at him waiting for his reaction. He was asked whether his wife Joan should be informed of her diagnosis. Andrew asked for 
Diagnostic Disclosure -Initial Investigative Phase

Establish Early Diagnosis\& Encourage patients to involve their family in the disclosure Understand attitudes of patient\& Family towards cognitive loss Discuss diagnostic uncertainty if any Address fears,concerns and their beliefs about dementia

\begin{tabular}{|c|} 
During Diagnosis \\
\hline Discuss it in a manner consistent with the patients' wishes \\
Use language which patient easily understands \\
Keep space for emotions, reflection \& tackle denial. \\
Additional information on Further tests, Finances, driving \\
Individual prognosis, Type of dementia and medications.
\end{tabular}

\section{Patient centered approach}

Use individualised approach taking into account cultural background. Look into patients expectations Assess immediate psychological impact.

Post diagnostic counselling should focus on remaining capabilities, coping strategies.

Fig. 1. Evidence based approach for diagnostic disclosure of Dementia.

advice from the doctor who informed him that it was ultimately his decision. Andrew decided to call Joan in the clinic room as he thought that Joan was a mature lady and would understand and adapt to the situation. Andrew felt he could perhaps help her realise that she did have a progressive memory problem. Andrew was informed of the diagnosis and she sat motionless, disbelieving in the diagnosis. For a while after the diagnosis was disclosed Joan was calm. She however had frequent appointments in the memory clinic with further tests due to which she began to rebel. Andrew helped her to go to a local day centre which worked for a while after which she refused to go. Joan started developing verbally aggressive behaviour towards Andrew and he bore the brunt of it. He was hence put in touch with the admiral nurse service for carer distress and is trying very hard to cope.

Case study 1 highlights several ethical problems, examples of bad clinical practice and is not the best example of patient centred care. Early diagnosis is beneficial and helpful (Milne\& Wilkinson, 2002). It helps patients to prepare and plan for the future. People with dementia want early disclosure of the diagnosis (Jha etal, 2001). People have the right to be informed of their diagnosis and this should not be withheld on the grounds that patient has dementia or memory problems. In the case study 1 the patient Joan suffered from lapses of memory for several years which went unnoticed. Early or even timely diagnosis would have helped the patient to plan for her future care and treatment. Patients' autonomy should be respected and patient should be informed of the diagnosis and then at the same time encouraged to share the diagnosis with their family and carer. In Case 1 Joan's autonomy 
was not respected and instead the patient's husband was informed of the diagnosis first and asked if the diagnosis can be broken to Joan when in fact Joan was capable of understanding the information. This also amounts to breach in confidentiality. In any memory service, patients' right to be informed of their diagnosis should be respected and diagnosis should not be withheld solely on grounds that it may provoke anxiety and suffering. Confidentiality should be maintained and if the patient clearly refuses for a disclosure this should be respected. Diagnosis should be a process, a series of steps (Aminzadeh etal, 2007) which was not the case in Case study1 where it was a sudden event. Diagnosis should be disclosed in a compassionate manner and should involve the patient maintaining dignity and a sense of hope (Connel $C$ etal, 2004). This did not happen in case study1 where the disclosure was insensitive and not person-centred. Dignity and solidarity were not maintained. Since the users and carers are quite vulnerable during this process, it is quite important to have space for emotions and reflection (Derksen E et al, 2006). Information on the illness, prognosis and necessary services was not provided in Case study 1 which is morally wrong. Giving clear and factual information to the patient in different forms is necessary. Post diagnostic counselling to focus on the remaining capability of the patient was not in place. This needs to be tailored specifically to the needs of the patient. (Derksen E etal, 2006).

\section{Ethical issues.}

Several ethical issues stand out in Case 1 however the second point is quite important.

1. Early diagnosis was not made

2. Patient's autonomy was not respected as carer was informed of the diagnosis prior to patient.

3. Confidentiality was breached.

4. Disclosure was sudden, insensitive and not person-centred.

5. No information and support was given after diagnostic disclosure to both patient and carer.

In Case study 1 it was ethically wrong that the carer was informed of the diagnosis first and asked if the diagnosis should be disclosed to the patient. Ethics dictates that a doctor should give an honest response about the diagnosis, even if it is uncertain to respect the patient's wellbeing and autonomy. Joan appears to have capacity to absorb the relevant information and hence is autonomous. The doctor should have respected Joan's autonomy and should have disclosed the diagnosis to her. After this disclosure the doctor should have taken Joan's consent to disclose the diagnosis to her husband. This ethical dilemma is commonplace in clinical practice and should be considered carefully. Clinicians are faced with a dilemma of respecting patient's autonomy on one hand and concerns of carers on the other hand. Nuffield Council of bioethics suggests a broad concept of autonomy called "Relational Autonomy" for patients suffering from Dementia. It suggests that as patient's sense of self is grounded in their social network, the whole family should be helped to support the autonomous wishes of the patient. This is a very useful concept and should be practiced.

\subsection{Case Study 2}

Elizabeth is a married lady in her early sixties who lives with her husband . Elizabeth works as a school teacher and teaches classical languages. Her work colleagues recently noticed some minor word finding problems which they found unusual taking into account her 
command on languages. Her husband noticed this too but put it down to anxiety as their son recently met with an accident and was in hospital due to which she has become somewhat distractable. They hence went to see the G.P. on insistence of their daughter . Her husband confirms that her word finding problems have not deteriorated but has noticed that Elizabeth avoids using the phone. Her husband also noticed that she can drift off from conversations but tends to get back to the original point. She on occasions can go to the kitchen and forget the very purpose of it and at times can mislay her personal belongings.

Her G.P carried out blood investigations and CT scan which were normal. She scored 28/30 on the MMSE. Her G.P. made a referral to Old Age Psychiatric services as he was unsure if Elizabeth was developing memory problems or if this was related to anxiety due to her son's accident.

The G.P referral was discussed in the Old age Psychiatry referral meeting and was allocated to be seen by the Neuropsychologist. An MRI with a medial temporal view was also ordered by the Consultant. The MRI sequences were discussed with the Neuroradiologist and agreed by him.

Elizabeth saw the Neuropsychologist in 2 weeks time for a Neuropsychological assessment which revealed early signs of cognitive impairment. She also had a MRI scan the same week which showed advanced involutional changes with both central and peripheral atrophy along with moderate bilateral hippocampal atrophy. She saw the Consultant the following week in his clinic. Elizabeth and her husband were called in together in the clinic. They were made comfortable and the purpose of the consultation was discussed. Elizabeth was asked if she wanted to know of her diagnosis and if she was happy to share it with her husband. After obtaining her consent, she was asked what she thought about her word-finding problems and what did she put it down to. She was given space to voice her concerns and her thoughts on the matter. She was then given a detailed feedback of the neuropsychological test she had last week and the findings were explained to her. She was then asked if she would like to see the MRI images on the computer and after taking her consent. The MRI scan findings were explained to her visually on the computer. Following this the diagnosis of dementia was broken in a very sensitive manner giving reasons as to why this was a disease process rather than normal ageing or a anxiety disorder. Elizabeth was given space for her emotions following which she was given a choice about the available medications. She was given patient information leaflets about local services . Patient was asked if she would like the letter copied to her and was given a choice about attending the post diagnostic counselling group and the Living Well Programme.

Discussion: This is a very good example of diagnostic disclosure and also how medical technology assists in speedy and early diagnosis. The G.P was quite candid that he was not able to come to grips of what was going on as so often is the case for Mild cognitive impairment and hence referred Elizabeth to old age psychiatric services. The expertise of the Consultant was very valuable as this patient required higher investigations ie MRI scan with specific sequences and also a Neuropsychological assessment.

The patient pathway was effective and the waiting time was reasonable. This did not cause undue distress to the patient or her family. The consultation was good and it was clear that he clinician breaking the diagnosis was experienced and did the diagnostic disclosure in a patient centred manner.

His communication skills were good and he did give space for emotions which is very important. MRI Brain images were shown which is a very powerful tool useful in diagnostic disclosure and also for tackling denial if any. 


\section{Conclusion}

Disclosure of diagnosis of dementia is not straightforward. It involves not only the patient but multiple people, professionals and family members. There is robust evidence which favours diagnostic disclosure. Most studies indicate that patients would like to be told of their diagnosis and would like their carers to be informed as well. Patients who are autonomous have the right to be informed of their diagnosis and doctors should try to involve family members. The new concept of Relational Autonomy is quite useful and hence should be practiced widely. In my view each case should be considered individually, choices should be respected irrespective of the dementia stage and disclosure should be an ongoing process. Questions still remain as to how much information should be disclosed in patients with advanced dementia who are not capable to understand the information. How do we balance autonomy on one hand and carers wishes not to disclose the diagnosis? Is disclosure an absolute right or a relative one?

\section{References}

Aminzadeh, F., Byszewski, A.,Molnar, F; Eisner ,M. Emotional impact of dementia diagnosis (2007) Exploring patients with dementia and caregivers' perspective Ageing Mental Health ;11:281-90

Bamford, C., Lamont, S., Eccles .M., Robinson L., May C., Bond, J. Disclosing a diagnosis of dementia: a systematic review (2004) Int J Geriatric Psychiatry.Feb; 19(2):151-69.

Carpenter, B., Dave, J. Gerontologist. 2004 Disclosing a dementia diagnosis: a review of opinion and practice, and a proposed research agenda Apr; 44(2):149-58.

Connell, C., Boise, L., Stuckey, J., Holmes, S., Hudson, M. Attitudes towards diagnosis and disclosure of dementia among family caregivers and primary care physicians Gerontologist 2004, 44: 500-7

DeCarli,C. et al.Qualitative estimates of medial temporal atrophy as a predictor of progression from mild cognitive impairment to dementia.Arch.Neurol.64,108115(2007)

Derksen. E., Vernooij-Dassen, M., Gillissen, F., Olde Rikkert, M., Scheltens, P. Aging Ment Health. ( 2006) Impact of diagnostic disclosure in dementia on patients and carers: qualitative case series analysis. September; 10

Downs, M., Clibbens, R., Rae, C. What do general practitioners tell people with dementia and their families about the condition? (2002)A survey of experiences in Scotland. Dementia: The International J. Soc. Res. Prac. 1: 47- 58

Drickamer, M., Lachs, M., (1992) should patients with Alzheimer's disease be told of their diagnosis? New England Journal of Medicine 1992; 326:947-51.

Duara,R. et al.Medial temporal lobe atrophy on MRI scans and the diagnosis of Alzheimer's disease .Neurology 71,1986-1992(2008

Georges J\& Gove D(2007) Disclosing a diagnosis; The Alzheimer's Europe View, Journal of Dementia Care, 15(6) Nov-Dec 28-30.

Glosser, G., Wexler, D., Balmelli, M., (1985) J Am Geriatric Soc. Physicians' and families' perspectives on the medical management of dementia. Jun; 33(6):383-91.

Gordon, M., Goldstein, D., Canadian Family Physician. (2001) Alzheimer's disease. To tell or not to tell. Sep; 47:1803-6, 1809 
Beady \& Gilleard (2002) The Person with Alzhemier's disease ,Pathways to understanding the experience Editor Harris, Braudy, Johns Hopkins University Press ,USA page 3-28

Knapp, M., Prince, M., Albanese ,E., Banerjee ,S., Dhanasiri ,S., Fernandez ,JL., Ferri ,C., McCrone,P .,Snell, T., Stewart,,R (2007), Dementia UK, The full report Alzhiemer's Society London

Pinner, G., Bouman, W (2002) to tell or not to tell: on disclosing the diagnosis of dementia. Int Psychogeriatrics 14:127-37

Pratt R\& Wilkinson H, (2001) Tell me the truth, The effect of being told the diagnosis of dementia from the perspective of the person with dementia. Mental Health foundation www.mentalhealth.org.uk

Jha, A., Tabet, N., Orrell, M. Int J Geriatr Psychiatry. (2001)To tell or not to tell-comparison of older patients' reaction to their diagnosis of dementia and depression Sep; 16(9):879-85

Maranski M. would you like to know what is wrong with you? (2000) on telling the truth to patients with dementia. Journal of medical ethics 26: 108-13

McKhann, G., Drachman, D., Folstein, M Neurology. (1984) Clinical diagnosis of Alzheimer's disease: Report of the NINCDS-ADRDA Work Group under the auspices of Department of Health and Human Services Task Force on Alzheimer's Disease

Novack, DH., Plumer, R., Smith R, L., Ochitill, H., Morrow G, R., Bennett, JM. JAMA. (1979) Changes in physicians' attitudes toward telling the cancer patient. Mar 2; 241(9):897-900.

Oken, D (1961) what to tell cancer patients. Journal of the American Medical Association, 175, 1120-1128

Rothlind, J \& Brandt J, Journal of Neuropsychiatry \& Clinical Neurosciences (1993) A brief assessment of frontal and subcortical functions in Dementia. 5: 73-77

Van Hout, HP. Vernooij-Dassen, MJ., Jansen DA., Stalman, WA. Aging Ment Health. (2006) Do general practitioners disclose correct information to their patients suspected of dementia and their caregivers? A prospective observational study. Mar; 10(2):151-5

Vassilas, C, Donaldson J (1998) Telling the truth: What General Practitioners say to patients with Dementia or terminal Cancer? British Journal of general practice March 48(428) 1081-1082 


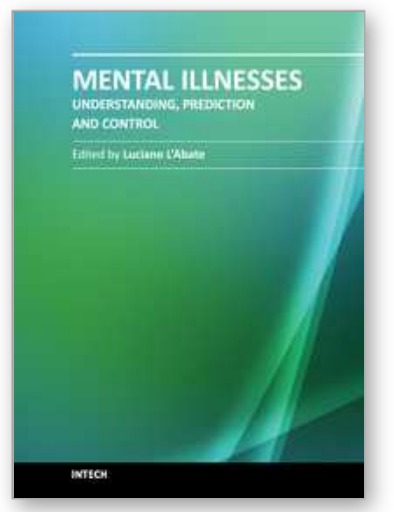

\author{
Mental IIInesses - Understanding, Prediction and Control \\ Edited by Prof. Luciano LAbate
}

ISBN 978-953-307-662-1

Hard cover, 458 pages

Publisher InTech

Published online 05, January, 2012

Published in print edition January, 2012

In the book "Mental Illnesses - Understanding, Prediction and Control" attention is devoted to the many background factors that are present in understanding public attitudes, immigration, stigma, and competencies surrounding mental illness. Various etiological and pathogenic factors, starting with adhesion molecules at one level and ending with abuse and maltreatment in childhood and youth at another level that are related to mental illness, include personality disorders that sit between mental health and illness. If we really understand the nature of mental illness then we should be able to not only predict but perhaps even to control it irrespective of the type of mental illness in question but also the degree of severity of the illness in order to allow us to predict their long-term outcome and begin to reduce its influence and costs to society. How can we integrate theory, research evidence, and specific ways to deal with mental illness? An attempt will be made in the last conclusive chapter of this volume.

\title{
How to reference
}

In order to correctly reference this scholarly work, feel free to copy and paste the following:

M. Dalvi (2012). Breaking a Diagnosis of Dementia, Mental Illnesses - Understanding, Prediction and Control, Prof. Luciano LAbate (Ed.), ISBN: 978-953-307-662-1, InTech, Available from:

http://www.intechopen.com/books/mental-illnesses-understanding-prediction-and-control/breaking-adiagnosis-of-dementia

\section{INTECH}

open science | open minds

\section{InTech Europe}

University Campus STeP Ri

Slavka Krautzeka 83/A

51000 Rijeka, Croatia

Phone: +385 (51) 770447

Fax: +385 (51) 686166

www.intechopen.com

\section{InTech China}

Unit 405, Office Block, Hotel Equatorial Shanghai

No.65, Yan An Road (West), Shanghai, 200040, China

中国上海市延安西路65号上海国际贵都大饭店办公楼 405 单元

Phone: +86-21-62489820

Fax: $+86-21-62489821$ 
(C) 2012 The Author(s). Licensee IntechOpen. This is an open access article distributed under the terms of the Creative Commons Attribution 3.0 License, which permits unrestricted use, distribution, and reproduction in any medium, provided the original work is properly cited. 